\title{
Dyskinetic Storm Induced by Intra-Operative Deep Brain Stimulator Placement
}

\author{
Amanda K. Hooper*, ${ }^{*}$, Tina M. Ellis ${ }^{1}$, Kelly D. Foote ${ }^{2}$, Pamela Zeilman ${ }^{1}$ and Michael S. Okun ${ }^{1,2}$ \\ ${ }^{I}$ University of Florida Department of Neurology, Movement Disorders Center, McKnight Brain Institute, USA \\ ${ }^{2}$ University of Florida Department of Neurosurgery, Movement Disorders Center, McKnight Brain Institute, USA
}

\begin{abstract}
Transient dyskinesia induced by deep brain stimulation (DBS) for Parkinson's disease (PD) has previously been reported as an intra-operative side effect usually indicating excellent lead placement and a positive outcome. Generally, stimulation-induced dyskinesia occurs contralateral to the side of stimulation. We report a case in which a patient developed a severe bilateral "dyskinetic storm" upon placement of the cannula and prior to macrostimulation during DBS surgery of the left subthalamic nucleus. This case illustrates that rarely dyskinetic storms may occur intra-operatively and become generalized.
\end{abstract}

\section{INTRODUCTION}

Dyskinesia is commonly associated with the long-term usage of medications to treat Parkinson's disease (PD), especially levodopa [1-3]. Deep brain stimulation (DBS) is one of the most efficacious treatments for medication refractory dyskinesia [4-7]. Both subthalamic nucleus (STN) DBS and globus palladus interna (GPi) DBS may induce dyskinesias in the intra-operative setting and in the "on" DBS condition $[8,9]$. Less commonly, the placement of the DBS electrode itself prior to test stimulation results in dyskinesia [10]. We report a case of a severe dyskinetic storm that began unilaterally and progressed rapidly to become generalized. The episode was unique in that it occurred prior to any test stimulation.

\section{CASE REPORT}

A 50-year-old man with a 17-year history of PD presented for medication refractory tremor, rigidity, bradykinesia, gait disorder, and severe on-off fluctuations associated with dyskinesia. His first symptom was a slight resting tremor in his right upper extremity. He initially took carbidopa/levodopa and trihexyphenidyl which led to an almost complete abatement of symptoms. However, his symptoms progressed and he required carbidopa/levodopa 0.5-2 tablets every 2 hours. Despite multiple medication adjustments and classes of medications, he experienced severe on-off fluctuations and half or more of his day was spent with disabling dyskinesia. Preoperatively his Unified Parkinson Disease Rating Scale (UPDRS) motor score improved from 81 off medication to 45 on medication.

During DBS surgery, he was placed in the recumbent position on the operating room table with his head fixed. A stereotactic CT-MRI fusion and multiple pass microelectrode mapping was used to identify the surgical target [11]. He

\footnotetext{
*Address correspondence to this author at the University of Florida Department of Neurology, Movement Disorders Center, McKnight Brain Institute, 704 SW $16^{\text {th }}$ Ave., Apt 302, Gainesville, FL 32601, USA; Tel: 615-330-
} 0133; Fax: 352-273-5575; E-mail: akhooper@ufl.edu was off medication for 20 hours at the start of the case, and 22.5 hours following lead placement at the end of the case. After recording and identification of the STN target, a modified UPDRS was performed and then a DBS electrode was inserted through a guide cannula (which was advanced to the target and then retracted) into the physiologically refined target. Prior to lead placement, no dyskinesia was observed. A slight dyskinesia was seen almost immediately in the right upper extremity. While performing a modified UPDRS, the dyskinesia worsened in the right upper extremity, spread to the right lower extremity, and then was observed in all extremities (see video). The patient's mental status was tested, and he was completely alert and oriented and indicated that this was a typical bout of severe dyskinesia. He then quickly progressed into a "dyskinetic storm," requiring multiple staff to hold him in position and to protect the integrity of the head ring. Propofol at a dose of $180 \mathrm{mg}$ (in four boluses of $40 \mathrm{mg}, 60 \mathrm{mg}$, $40 \mathrm{mg}$, and $40 \mathrm{mg}$ ) was required to sedate him and control the dyskinesia. Each DBS contact 0-3 was tested at $135 \mathrm{~Hz}$ and a pulse width of $90 \mathrm{~ms}$; however, it was difficult to discern any worsening of symptoms or to segregate side effects because of the severity of the dyskinesia. Dyskinesia was not worsened by DBS; however, because of its severity, the examination was difficult. In recovery, no further dyskinesia was noted. He stayed in the hospital overnight. The patient's levodopa was held overnight and he returned to his normal dose of medication the next morning and was discharged without incident. During the first 30 days post-operatively, the patient reported dyskinesia at a similar level to his dyskinesia prior to surgery. The patient had a second DBS surgery to implant the opposite STN six months after his first implantation without incident. Following the first DBS surgery, he had stimulation induced dyskinesias during programming and then chronically, but they mostly abated with slight medication and programming adjustments (he complained of mild dyskinesias mainly late in the day). Following his second DBS (right STN) he reduced his carbidopa/levodopa further to 1 tablet at each dose and spread the interval to every four hours. Six months following his second DBS he reported only very rare non-daily dyski- 
nesia (UPDRS off medication on stimulation score of 32; and on medication on stimulation score of 28).

\section{DISCUSSION}

Intra-operative dyskinesia is considered by most expert groups to be a positive prognostic indicator of the success of DBS surgery; however, level-one evidence for this observation has not been established. Further, most intra-operative dyskinesia is unilateral and induced by stimulation through the DBS device. Our case is unique in that the dyskinesia was induced by collision of the guide cannula/lead placement. Another distinguishing feature was the spread from unilateral to bilateral dyskinesia and finally to a generalized dyskinetic storm. This patient also notably had a benign post-operative course and eventual resolution of dyskinesia despite some early stimulation induced dyskinesia.

Previous reports of stimulation-induced dyskinesia or lesion-induced dyskinesia exist in the literature. In GPi DBS or STN DBS, the onset of dyskinesia (usually on the contralateral side) has often been observed during test stimulation and also commonly occurs within a few seconds of activation [9, 12]. In many cases, especially STN DBS or STN lesions, there is a later onset of dyskinesia or hemiballism, which may resolve over time [8, 10, 12-18]. Studies in Parkinsonian rats and primates have also shown dyskinesia with STN DBS [19, 20]. Stimulation-induced dyskinesias have been reported to worsen with increased voltage and improve when the amplitude of stimulation is reduced [10, $12,15,21]$. Uncharacteristically, our patient experienced severe dyskinesia before any stimulation was applied. The dyskinesia induced by stimulation or lesion often resembles the patient's previous medication-induced dyskinesia, but has also been reported to be milder [12]. The patient presented in this report did have severe levodopa-induced baseline dyskinesia prior to surgery, which may have placed him at risk for the intra-operative dyskinetic storm. It could be argued that in our case, the cannula placement induced a lesion-like effect and resulted in severe dyskinesia. There may have been microhemorrhage or other lesion-like effects that exacerbated the severe symptoms. These explanations, however, fail to account for the generalized dyskinetic storm that was observed, and post-operative CT did not reveal hemorrhage. The rapidity of his recovery post-operatively and the resolution of the storm argue against a typical lesion effect. It is conceivable that there are unilateral as well as bilateral dyskinetic pathways that may be disrupted even with a unilateral procedure [22], but further research will be needed clarify these points.

Although we believe from our own experience that the presence of dyskinesia is a good prognostic indicator, more studies will need to be done to establish this as a predictive factor. Other authors have commented on the positive outcomes in these patients $[5,7,9,23,24]$; however, there is currently no significant evidence in the literature showing the prognostic value of intraoperative DBS-induced dyskinesia This case illustrates that rarely dyskinetic storms may also occur bilaterally and intra-operatively even without stimulation. It is important to act quickly if this complication occurs in order to preserve the safety of the patient and the placement of the DBS lead.

\section{SUPPLEMENTARY MATERIAL}

This article also contain supplementary material (video) and it can be viewed at www.bentham.org/open/toneurosj

\section{ACKNOWLEDGEMENTS}

We acknowledge the support of the National Parkinson Foundation Center of Excellence.

\section{REFERENCES}

[1] Fabbrini G, Brotchie JM, Grandas F, Nomoto M, Goetz CG. Levodopa-induced dyskinesias. Mov Disord 2007; 22(10): 137989.

[2] Thanvi B, Lo N, Robinson T. Levodopa-induced dyskinesia in Parkinson's disease: clinical features, pathogenesis, prevention and treatment. Postgraduate Med J 2007; 83(980): 384-8.

[3] Zesiewicz TA, Sullivan KL, Hauser RA. Levodopa-induced Dyskinesia in Parkinson's Disease: epidemiology, Etiology, and Treatment. Curr Neurol Neurosci Rep 2007; 7(4): 302-10.

[4] The Deep-Brain Stimulation for Pakinson's Disease Study Group. Deep-brain stimulation of the subthalamic nucleus or the pars interna of the globus pallidus in Parkinson's disease. N Engl J Med 2001; 345(13): 956-63.

[5] Benabid AL, Benazzouz A, Limousin P, et al. Dyskinesias and the subthalamic nucleus. Ann Neurol 2000; 47(4 Suppl 1): S189-92.

[6] Fraix V, Pollak P, Van Blercom N, et al. Effect of subthalamic nucleus stimulation on levodopa-induced dyskinesia in Parkinson's disease. 2000. Neurology 2001; 57(10 Suppl 3): S60-2.

[7] Halpern C, Hurtig H, Jaggi J, Grossman M, Won M, Baltuch G. Deep brain stimulation in neurologic disorders. Parkinsonism related Disord 2007; 13(1): 1-16.

[8] Alvarez L, Macias R, Lopez G, et al. Bilateral subthalamotomy in Parkinson's disease: initial and long-term response. Brain 2005; 128(Pt 3): 570-83.

[9] Houeto JL, Welter ML, Bejjani PB, et al. Subthalamic stimulation in Parkinson disease: intraoperative predictive factors. Arch Neurol 2003; 60(5): 690-4.

[10] Herzog J, Pinsker M, Wasner M, et al. Stimulation of subthalamic fibre tracts reduces dyskinesias in STN-DBS. Mov Disord 2007; 22(5): 679-84.

[11] Okun MS, Vitek JL. Lesion therapy for Parkinson's disease and other movement disorders: update and controversies. Mov Disord 2004; 19(4): 375-89.

[12] Kumar R. Methods for programming and patient management with deep brain stimulation of the globus pallidus for the treatment of advanced Parkinson's disease and dystonia. Mov Disord 2002; 17 (Suppl 3): S198-207.

[13] Chen CC, Lee ST, Wu T, Chen CJ, Huang CC, Lu CS. Hemiballism after subthalamotomy in patients with Parkinson's disease: report of 2 cases. Mov Disord 2002; 17(6): 1367-71.

[14] Doshi P, Bhatt M. Hemiballism during subthalamic nucleus lesioning. Mov Disord 2002; 17(4): 848-9.

[15] Krack P, Batir A, Van Blercom N, et al. Five-year follow-up of bilateral stimulation of the subthalamic nucleus in advanced Parkinson's disease. N Engl J Med 2003; 349(20): 1925-34.

[16] Merello M, Perez-Lloret S, Antico J, Obeso JA. Dyskinesias induced by subthalamotomy in Parkinson's disease are unresponsive to amantadine. J Neurol Neurosurg Psychiatry 2006; 77(2): 172-4.

[17] Tseng HM, Su PC, Liu HM. Persistent hemiballism after subthalamotomy: the size of the lesion matters more than the location. Mov Disord 2003; 18(10): 1209-11.

[18] Su PC, Tseng HM, Liu HM, Yen RF, Liou HH. Treatment of advanced Parkinson's disease by subthalamotomy: one-year results. Mov Disord 2003; 18(5): 531-8.

[19] Boulet S, Lacombe E, Carcenac C, et al. Subthalamic stimulationinduced forelimb dyskinesias are linked to an increase in glutamate levels in the substantia nigra pars reticulata. J Neurosci 2006; 26(42): 10768-76.

[20] Guridi J, Herrero MT, Luquin MR, et al. Subthalamotomy in parkinsonian monkeys. Behavioural and biochemical analysis. Brain 1996; 119 ( Pt 5): 1717-27.

[21] Thobois S, Mertens P, Guenot M, et al. Subthalamic nucleus stimulation in Parkinson's disease: clinical evaluation of 18 patients. J Neurol 2002; 249(5): 529-34. 
[22] Chung SJ, Jeon SR, Kim SR, Sung YH, Lee MC. Bilateral effects of unilateral subthalamic nucleus deep brain stimulation in advanced Parkinson's disease. Eur Neurol 2006; 56(2): 127-32.

[23] Krack P, Fraix V, Mendes A, Benabid AL, Pollak P. Postoperative management of subthalamic nucleus stimulation for Parkinson's disease. Mov Disord 2002; 17 (Suppl 3): S188-97.
[24]

Pollak P, Krack P, Fraix V, et al. Intraoperative micro- and macrostimulation of the subthalamic nucleus in Parkinson's disease. Mov Disord 2002; 17(Suppl 3): S155-61.

(C) Hooper et al.; Licensee Bentham Open.

This is an open access article licensed under the terms of the Creative Commons Attribution Non-Commercial License (http://creativecommons.org/licenses/by-nc/3.0/) which permits unrestricted, non-commercial use, distribution and reproduction in any medium, provided the work is properly cited. 